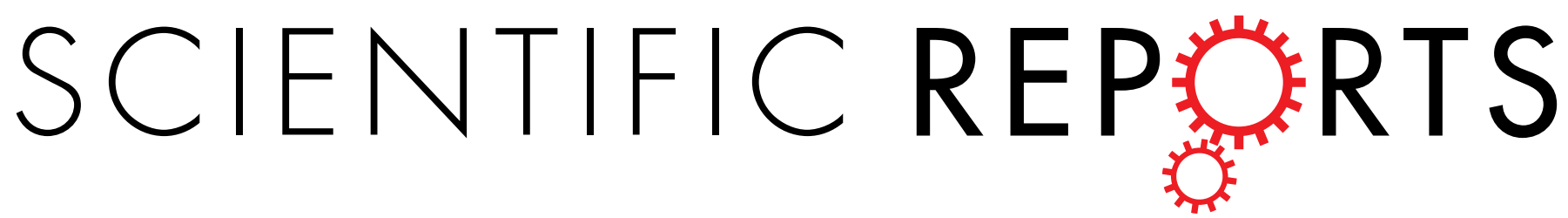

\title{
Corrigendum: An embryo of protocells: The capsule of graphene with selective ion channels
}

Zhan Li, Chunmei Wang, Longlong Tian, Jing Bai, Huijun Yao, Yang Zhao, Xin Zhang, Shiwei Cao, Wei Qi, Suomin Wang, Keliang Shi, Youwen Xu, Zhang Mingliang, Bo Liu, Hongdeng Qiu, Jie Liu, Wangsuo Wu, Xiaoli Wang \& An Wenzhen

Scientific Reports 5:10258; doi: 10.1038/srep10258; published online 19 May 2015; updated on 31 July 2015

This Article contains an error in the affiliation of Jie Liu. The correct affiliation is listed below: Institute of Modern Physics, Chinese Academy of Sciences, Lanzhou, 730000, P.R. China 